\title{
Propagation of radio waves in pulsar magnetospheres
}

\author{
S.A. Petrova \\ Institute of Radio Astronomy, \& Chervonopraporna Str., Kharkiv, \\ 310002, Ukraine \\ Yu.E. Lyubarskii \\ Institute of Radio Astronomy, \& Chervonopraporna Str., Kharkiv, \\ 310002, Ukraine
}

\begin{abstract}
The morphological classification of pulsar profiles is interpreted within the frame of the primordial hollow-cone emission model taking into account wave propagation in the magnetosphere. In this context the frequency evolution of the profiles as well as the difference in polarization properties of core and conal components can be explained naturally.
\end{abstract}

Pulsar magnetospheres are filled with an ultrarelativistic electron-positron plasma streaming along the open field lines of the dipolar magnetic field. Since radio emission is believed to originate well within the magnetosphere, the characteristics of the observed pulsar radiation should be conditioned by wave propagation in the magnetospheric plasma. The waves are expected to originate at frequencies

$$
\omega \sim \omega_{p} \sqrt{\gamma}
$$

where $\omega_{p}$ is the customary plasma frequency, $\gamma$ the Lorentz-factor. The point is that the waves excited by the plasma instability acquire the frequencies (1) in the course of induced scattering, which is rather efficient at pulsar conditions (Lyubarskii 1996). According to the standard models, the plasma density beyond the tube edge as well as near the magnetic axis is negligible and, correspondingly, it should decrease towards both the inner and outer tube edges.

Refraction of radio waves in pulsar plasma turns out to be significant. Ray deviation is found to be chiefly determined by the plasma density gradient across the tube. Obviously, the rays deflect in the direction of plasma density decrease. Hence, the rays emitted near the inner boundary of the plasma flow deviate towards the magnetic axis and can be recognized as core emission, whereas those emitted near the outer tube edge deviate away from the magnetic axis and form conal outriders (Petrova \& Lyubarskii 1999). Refraction of rays in the open field line tube turns out to be frequency-dependent. Both transverse density gradient and emitted frequency decrease with the distance from the neutron star. So at lower frequencies refraction is less efficient and the separation of the profile components is less prominent. This is quite what is observed (Rankin 1983a).

Refraction of rays in the region of plasma density decrease toward the outer tube edge leads to the increase of pulse width. At high frequencies the pulse 
width is determined by refraction and remains nearly constant. At low frequencies it is determined by the width of the open field line tube and therefore increases with the wavelength. If the pulse width is formed by the rays emitted in the region of plasma density decrease toward the inner tube edge, its frequency evolution is essentially different (Lyubarskii \& Petrova 1998). At sufficiently high frequencies the rays progressively deviate toward the magnetic axis and ultimately begin to intersect it. Then the pulse width increases with the frequency. At still higher frequencies the rays have time to turn round and start deviation in the opposite direction, so that the pulse width decreases again. Note that both types of the frequency dependence of pulse width are really observed in pulsars (Rankin 1983b).

As the waves are propagating through the magnetosphere their polarization also evolves essentially. The plasma embedded in a superstrong magnetic field allows two linearly polarized normal waves. Wave mode coupling in the polarization-limiting region gives rise to circular polarization in outgoing radiation (Cheng \& Ruderman 1979). Note that the polarization-limiting effect occurs on condition that the $\mathbf{k} \times \mathbf{b}$-plane rotates along the wave trajectory. The ray can deviate from the initial magnetic line plane either because of magnetosphere rotation or on account of ray refraction given the nonaxisymmetric plasma density distribution. The wave mode coupling introduced by magnetosphere rotation results in the circular polarization of one sense throughout the pulse (Cheng \& Ruderman 1979). The wave mode coupling because of refraction leads to circular polarization with the sense reversal near the pulse centre (Petrova \& Lyubarskii 1999). At high frequencies, which originate at low altitudes, refraction is strong and magnetosphere rotation is negligible. At lower frequencies refraction ceases, whereas rotational effect becomes essential. Hence, the frequency evolution of the circular polarization profile should be as follows. At high frequencies the profile is antisymmetric, the maximum circular polarization increasing with the frequency due to the increasing role of refraction. At lower frequencies the profile becomes symmetrical. This is just what is observed (Han, Manchester, \& Qiao 1999).

Significant departure of position angle swing from the common S-shape is another consequence of sufficiently strong polarization-limiting effect. Note that irregular position angle swing and large amounts of circular polarization are really observed together, both being the attributes of core emission (e.g. Rankin 1990).

\section{References}

Cheng, A.F. \& Ruderman, M.A. 1979, ApJ, 229, 348

Han, J.L., Manchester, R.N., \& Qiao, G.J. 1999, this volume

Lyubarskii, Yu.E. 1996, A\&A, 308, 809

Lyubarskii, Yu.E. \& Petrova, S.A. 1998, A\&A, 333, 181

Petrova, S.A. \& Lyubarskii, Yu.E. 1999, A\&A, in press

Rankin, J.M. 1983a, ApJ, 274, 333

Rankin, J.M. 1983b, ApJ, 274, 359

Rankin, J.M. 1990, ApJ, 352, 247 